\title{
T-overlap T-migrative Functions: A Generalization of Migrativity in t-Overlap Functions
}

\section{Funciones t-migrativas t-overlap: una generalización de migratividad en funciones t-overlap}

\author{
Funções T-sobrepostas T-sobrepostas: uma generalização da \\ migração para funções T-sobrepostas \\ Hugo Alain Zapata-Ceballos ${ }^{1}$
}

Humberto Bustince (iD) 2 Graçaliz Pereira Dimuro (iD) 3

Received: November 2019

Accepted: April 2020

How to cite: Zapata-Ceballos, H., Bustince, H., Dimuro, G.P. (2020). T-overlap T-migrative Functions: a generalization of migrativity in t-overlap functions. Revista Científica, 38(2). https://doi.org/10.14483/23448350.15601

\begin{abstract}
This paper introduces a generalization of migrative functions by extending the conditions of the product operation applied in the variables. We operate a number with the variables according to a t-norm instead of multiplying the variable $\mathrm{x}$ by this number. Such generalization, whenever it occurs, is called a t-migrative function with respect to such t-norm. Furthermore, we analyse the main properties of t-migrative and t-overlap functions. We introduce some interesting methods of construction of such functions.
\end{abstract}

Keywords: Migrative function, overlap function, t-norm.

\section{Resumen}

Este artículo introduce una generalización de funciones migrativas por extensión de la condición de la operación producto aplicada en las variables. Más específicamente, en lugar de exigir multiplicar la variable $x$ por un número real $\alpha$, en este trabajo se trabaja este número $\alpha$ con las variables de acuerdo a una t-norma. Se denomina a esta generalización función t-migrativa con respecto a tal t-norma. Luego se analizan las propiedades principales de funciones t-migrativas en funciones t-overlap y se introducen algunos métodos de construcción de este tipo de funciones.

Palabras clave: función migrativa, función overlap, normas triangulares.

\footnotetext{
${ }^{1}$ Fundación universitaria Los Libertadores, Universidad de Sucre. Colombia. hazapatac@libertadores.edu.co hugo.zapata@unisucre.edu.co

${ }^{2}$ Universidad Pública de Navarra. España. bustince@navarra.es

${ }^{3}$ Universidade Federal do Rio Grande, FURG, Brasil. gracaliz@furg.br
} 


\section{Resumo}

Este artigo apresenta uma generalização das funções migratórias estendendo as condições da operação do produto aplicada nas variv́eis. Mais especificamente, em vez de exigir a multiplicação da variv́el $x$ por um número real $\alpha$, neste trabalho operamos isso $\alpha$ número com as variáveis de acordo com a norma t. Chamamos essa generalização de uma função t-migrative em relação a essa norma t. Em seguida se analiza as principais propriedades das funções t-sobreposição migratórias e introduzimos alguns métodos de construoção.

Palavras-chaves: função migratória, função de sobreposição, norma $\mathrm{t}$.

\section{Introduction}

The purpose of this paper is to generalize the notion of migrative functions by relaxing one of the conditions of product operators applied in the variables. The notion of migrative function was introduced and studied in (Bustince, Montero and Mesiar, 2009). We follow a different approach. Rather than multiplying the variables of the function by a real number a, we operate this number with a t-norm.We allow it some kind of threshold, defined in terms of a t-norm $T$. We call such generalization a t-migrative function with respect to $T$ and here after, we apply the migrativity to t-overlap functions. We observed that, this simple generalization allows us to state several interesting properties, which can be applied in fuzzy rule-based systemto eliminated bad rules when computing the compatibility degree. Section 1 presents some preliminary concepts. In Section 2 , we propose some method of construction of such function. We also study the main properties of the generalized functions.

\section{Preliminaries}

\subsection{Triangular Norms}

One of the basic concepts of the fuzzy theory is triangular norms or t-norms. In this paper, these functions are frequently used. We begin with the basic definition of $t$-norms.

Definition 1 (See Bustince, Burillo, and Soria (2003)) A triangular norm or t-norm is an aggregation function $T:[0,1]^{2}-[0,1]$ such that:

(i) $T(x, 1)=x$ for all $x \in[0,1]$, (ii) $T(x, y) \leq T(z, u)$ if $x \leq z$ and $y \leq u$,

(iii) $T(x, y)=T(y, x)$ for all $x, y \in[0,1]$,

(iv) $T(T(x, y), z)=T(x, T(y, z))$ for all $x, y, z \in$ $[0,1]$.

We then present some examples of t-norms which are of great interest.

Example 1 The function $T:[0,1]^{2}-[0,1]$, defined by $T(x, y)=\min \{x, y\}$ is a t-norm.

Definition 2 A t-norm function is strict if it is strictly increasing for its two variables, that is to say if $x_{1}<x_{2}$ and $y \neq 0$ then $T\left(x_{1}, y\right)<T\left(x_{2}, y\right)$.

Definition 3 A t-norm function is positive if $T(x, y)=0$ iff $x y=0$.

\subsection{Migrativity}

The concept of $\alpha$-migrativity was introduced by Durante and Sarkoci (2008). A bivariate operation's class having a property previously presented by Mesiar and Novak (1996) which was later studied by Fodor and Rudas (2007).

Definition 4 (Durante and Sarkoci 2008) Let $\alpha \in[0,1]$ be fixed. A bivariate operation

$G:[0,1]^{2} \rightarrow[0,1]$ is $\alpha$-migrative if $G(\alpha x, y)=G(x, \alpha y)$, for all $x, y \in[0,1]$.

From the definition above, we can easily observed that all function $G:[0,1]^{2}-[0,1]$ is 1-migrative, as $G(x, y)=G(1 . x, y)=G(x, 1 . y)$. This definition is referred to as a predetermined $\alpha$. We generalized the concept of $\alpha$-migrativity the next form,

Definition 5 (See Bustince, Montero and Mesiar (2009)) A function $G:[0,1]^{2}-[0,1]$ is called migrative if and only if $G(\alpha x, y)=G(x, \alpha y)$ for all $x, y \in[0,1]$ and for all $\alpha \in[0,1]$.

Example 2 The function $h:[0,1]^{2}-[0,1]$, defined by $h(x, y)=x y$, is migrative.

Example 3 La function $G:[0,1]^{2}-[0,1]$, defined by $G(x, y)=\frac{x+y}{2}$, shows that

$$
\begin{aligned}
G(1 . x, y) & =\frac{1 . x+y}{2} \\
& =\frac{x+1 . y}{2} \\
& =G(x, 1 . y)
\end{aligned}
$$


thus $G$ is a 1-migrative function, but if you take $\alpha=\frac{1}{2}$, it shows that $G\left(\frac{1}{2} x, y\right) \neq G\left(x, \frac{1}{2} y\right)$. This is easy to see, in particular if it is done $x=1$ and $y=\frac{1}{2}$.

The following lemma is the characterization of the main migrative functions.

Lemma 1 (Bustince, et al., 2010) A function

$$
G:[0,1]^{2}-[0,1]
$$

is migrative if and only if $G(x, y)=G(1, x y)$, for all $x, y \in[0,1]$.

From the lemma (Bandler and Kohout,1980), we deduce the following corollary, in which a characterization of bivariate migrative function is seen as a function of a variable.

Corollary 1 (Bustince, et al., 2010) A function $G$ : $[0,1]^{2}-[0,1]$ is migrative if and only if ther exists a function $g:[0,1]-[0,1]$ such that $G(x, y)=g(x y)$ for all $x, y \in[0,1]$.

\subsection{Overlap and T-Overlap Functions}

In this section, we present the definition of overlap function as well as some of its properties. This type of functions constitutes one of the most important pillars in this work.

Definition 6 (Bustince, et al., 2010) A function $G_{S}$ : $[0,1]^{2}-[0,1]$ is an overlap function, if it satisfies the following conditions:

$\left(G_{s} 1\right) G_{s}$ is symmetrical,

$\left(G_{s} 2\right) G_{s}(x, y)=0$ if and only if $x y=0$,

$\left(G_{s} 3\right) G_{s}(x, y)=1$ if and only if $x y=1$,

$\left(G_{s} 4\right) G_{s}$ is not decreasing,

$\left(G_{s} 5\right) G_{s}$ is continuous.

Example 4 An example of an overlap function is the product function $h(x, y)=x y$, with $x, y \in[0,1]$.

Example 5 The function $G(x, y)=\sin \left(\frac{\pi}{2} x y\right)$ is an overlap function.

Example 6 Other example of overlap function is $G(x, y)=\tan \left(\frac{\pi}{4} x y\right)$.

Revista Científica • ISSN 0124-2253 • e-ISSN 2344-2350 • May-August • Bogotá-Colombia • N. 38(2) • pp. 184-189
The concept of the generalization of the overlap function, is obtained by changing the condition $\left(G_{s} 2\right)$ given in (Alcala-Fdez, Alcala and Herrera, 2011). This property requires that given an overlap function $G_{S}$, then $G_{S}(x, y)=0 \Leftrightarrow x y=0$. In this generalization, the product operation is replaced by a t-norm $T:[0,1]^{2}-[0,1]$.

Definition 7 Let $T:[0,1]^{2}-[0,1]$ be a t-norm. A function $G_{T}:[0,1]^{2}-[0,1]$ is said to be a t-overlap function with respect to $T$ if the following conditions holds:

$\left(G_{T} 1\right) G_{T}(x, y)=G_{T}(y, x)$,

$\left(G_{T} 2\right) G_{T}(x, y)=0 \Leftrightarrow T(x, y)=0$,

$\left(G_{T} 3\right) G_{T}(x, y)=1 \Leftrightarrow x=y=1$,

$\left(G_{T} 4\right) G_{T}$ is increasing,

$\left(G_{T} 5\right) G_{T}$ is continuous.

\section{T-Migrativity}

We then present a generalization of the migrativity concept where the multiplication operation is replaced by a t-norm.

Definition 8 A two-dimensional $G$ function is said to be $t$-migrative with respect to a $t$-norm $T$ if for all $\alpha \in[0,1]$ we have that $G(x, T(\alpha, y))=G(T(x, \alpha), y)$ for all $x, y \in$ $[0,1]$.

Traditionally, a migrativity property is given for the particular case in which $\alpha=0$.

Proposition 1 A function $G:[0,1]-[0,1]$ is 0 -tmigrative if and only if $G(x, 0)=G(0, y)$

Proof 1 If $G$ is 0 -t-migrative then $G(x, 0)=$ $G(x, T(0, y))=G(T(x, 0), y)=G(0, y)$ If $G(x, 0)=$ $G(0, y)$ then as for all t-norm $T(0, x)=0$ then $G(x, T(0, y))=G(T(x, 0), y)$.

The following theorem broadly generalizes theorem 1

Theorem 1 A function $G:[0,1]^{2}-[0,1]$ is t-migrative with respect to a t-norm $T$ if and only if there exists a function $g:[0,1]-[0,1]$ such that $G(x, y)=g(T(x, y))$.

Proof 2 Let $G(x, y)=g(T(x, y))$, then

$$
\begin{gathered}
G(x, T(y, z))=g(T(x, T(y, z)))=g(T(T(x, y), z))= \\
G(T(x, y), z) .
\end{gathered}
$$

If $G$ is t-migrative with respec to a $t$-norm $T$, then

$$
G(x, y)=G(T(x, 1), y)=G(1, T(x, y))
$$


for all $x, y \in[0,1]$, if $G(x, y)=G(u, v)$ then $G(1, T(x, y))=G(1, T(u, v))$ where $T(x, y)=T(u, v)$ thus $g$ is well defined

The next corollary is a generalization of corollary 1

Corollary $2 G$ is t-migrative with respec to a t-norm $T$ if and only if

$$
G(x, y)=G(1, T(x, y))
$$

The following corollary shows an obvious consequence of t-migrativity.

Corollary 3 If $G$ is $t$-migrative with respect to a $t$-norm $T$ then $G$ is symmetrical.

Proof $3 G(x, y)=G(1, T(x, y))=G(1, T(y, x))=$ $G(y, x)$.

Below is a list of some of the properties of t-migrative functions.

Theorem 2 Let $G:[0,1]^{2}-[0,1]$ be a t-migrative function, then:

1. $G$ is non-decreasing if and only if $g$ is nondecreasing.

2. $G$ is strictly increasing in $[0,1]^{2}$ if and only if $g$ and $T$ are strictly increasing.

3. $G(1,1)=1$ if and only if $g(1)=1$.

4. $G(0,0)$ if and only if $g(0)$.

5. $G$ is continuous if and only if $g$ and $T$ are continuous.

Proof 4 1. Suppose that $G$ is a non-decreasing function. Let $x, y \in[0,1]$ such that $x \leq y$, then $G(x, 1) \leq G(y, 1)$, thus $g(T(x, 1)) \leq g(T(y, 1))$, therefore $g(x) \leq g(y)$. Suppose that $g$ is a nondecreasing function and $x, y \in[0,1]$ such that $x \leq y$ then for all $z \in[0,1]$ is true that $T(x, z) \leq$ $T(y, z)$, therefore $g(T(x, z)) \leq g(T(y, z))$ thus $G(x, z) \leq G(y, z)$.

2. Analogous.

3. $G(1,1) \Leftrightarrow g(T(1,1))=1 \Leftrightarrow g(1)=1$

4. $G(0,0) \Leftrightarrow g(T(0,0))=0 \Leftrightarrow g(0)=0$

5. $G$ is continuous if and only if $g$ and $T$ are continuous.
One of the most important aspects of this work is the generalization of the migratives of overlap functions. One of the results of this generalization is shown.

Theorem 3 If $G_{T}$ is a t-overlap function with respect to the continuous t-norm $T$, then

$$
G(x, y)=G_{T}(1, T(x, y))
$$

is a t-overlap t-migrative function with respect to $T$.

\section{Proof 5 1. Evidently $G$ is Symmetrical.}

2. $G(x, y)=0 \Leftrightarrow G_{T}(1, T(x, y))=0 \Leftrightarrow T(x, y)=$ 0.

3. $G(x, y)=1 \Leftrightarrow G_{T}(1, T(x, y))=1 \Leftrightarrow T(x, y)=$ $1 \Leftrightarrow x=y=1$.

4. $G$ is continuous.

5. $G$ is no decreasing.

$G(T(x, y), z)=G_{T}(1, T(T(x, y), z))=$ $G_{T}(1, T(x, T(y, z)))=G(x, T(y, z))$.

The following theorem shows that the convex sum of t-overlap t-migrative functions with respect to a continuous t-norm is also t-overlap t-migrative function.

Theorem 4 If $\alpha_{i} \geq 0 \forall i=1,2, \ldots, n, \sum_{i=1}^{n} \alpha_{i}=1, G_{i}$ are overlap functions and $T$ is a t-norm continuous, then

$$
G(x, y)=\sum_{i=1}^{n} \alpha_{i} G_{i}(1, T(x, y))
$$

is t-overlap t-migrative function with respect to $T$.

\section{Proof 6 1. $G$ is symmetrical.}

2. $G(x, y)=0 \Leftrightarrow \sum_{i=1}^{n} \alpha_{i} G_{i}(1, T(x, y))=0 \Leftrightarrow$ $\alpha_{i} G_{i}(1, T(x, y))=0$. Given that $\sum_{i=1}^{n} \alpha_{i}=1$ and $\alpha_{i} \geq 0 \forall i=1,2, \ldots, n$ then exist $\alpha_{k} \neq 0$ thus if $\alpha_{k} G_{k}(1, T(x, y))=0$ then $G_{k}(1, T(x, y))=0 \Rightarrow$ $T(x, y)=0$. If $T(x, y)=0$. then $G_{i}(1, T(x, y))=$ 0 for all $i=1,2, \ldots, n$ thus $G(x, y)=0$.

3. $G(x, y)=1 \Leftrightarrow \sum_{i=1}^{n} \alpha_{i} G_{i}(1, T(x, y))=$ $1 \Leftrightarrow \sum_{i=1}^{n} \alpha_{i} G_{i}(1, T(x, y))=\sum_{i=1}^{n} \alpha_{i}$ thus $\sum_{i=1}^{n} \alpha_{i}\left(1-G_{i}(1, T(x, y))\right)=0$ then $\alpha_{i}(1-$ $\left.G_{i}(1, T(x, y))\right)=0$ for all $i=1,2, \ldots, n$ since $\alpha_{i} \geq 0 \forall i=1,2, \ldots, n$, and $\sum_{i=1}^{n} \alpha_{i}=1$, then exist $\alpha_{k} \neq 0$ thus if $\alpha_{k}\left(1-G_{k}(1, T(x, y))\right)=0$ then $1-G_{k}(1, T(x, y))=0 \Rightarrow G_{k}(1, T(x, y))=$ $1 \Rightarrow T(x, y)=1 \Rightarrow x=y=1$.

4. $G$ is continuous.

5. $G$ is non-decreasing.

Theorem 5 If $T_{1}$ and $T_{2}$ are continuous and $G_{T}$ is a overlap function, then

Revista Científica • ISSN 0124-2253 • e-ISSN 2344-2350 • May-August • Bogotá-Colombia • N. 38(2) • pp. 184-189 


$$
G(x, y)=G_{T}\left(T_{1}(x, y), T_{2}(x, y)\right)
$$

is a t-overlap t-migrative function with respect to $T_{1}$ or $T_{2}$.

\section{Proof 7 1. $G$ is symmetrical.}

2. $G(x, y)=0 \Leftrightarrow G_{T}\left(T_{1}(x, y), T_{2}(x, y)\right)=0 \Leftrightarrow$ $T_{1}(x, y)=0 \vee T_{2}(x, y)=0$.

3. $G(x, y)=1 \Leftrightarrow G_{T}\left(T_{1}(x, y), T_{2}(x, y)\right)=1 \Leftrightarrow$ $T_{1}(x, y)=1 \wedge T_{2}(x, y)=1 \Leftrightarrow x=y=1$.

4. $G$ is continuous.

5. $G$ is non-decreasing.

Corollary 4 If $G$ is given as in the previous theorem and $T_{1}=T_{2}$ then $G$ is t-migrative.

Proof $8 G(x, T(y, z))=G_{T}\left(T_{1}(x, T(y, z)), T_{2}(x, T(y, z))\right)=$ $G_{T}\left(T_{1}(T(x, y), z), T_{2}(T(x, y), z)\right)=G(T(x, y), z)$.

Corollary 5 If $T$ is a t-norm and a strong negation $n$, then

$$
G(x, y)=\frac{T(x, y)}{T(x, y)+n T(x, y)}
$$

is a $t$-overlap t-migrative function with respect to $T$.

Theorem 6 If $G_{T}$ is an overlap function and $T$ is a continuous t-norm, then

$$
G(x, y)=2^{G(1, T(x, y))}-1
$$

is a t-overlap t-migrative function.

\section{Proof 9 1. $G$ is symmetrical.}

2. $G(x, y)=0 \Leftrightarrow 2^{G(1, T(x, y))}-1=0 \Leftrightarrow$ $2^{G(1, T(x, y))}=1 \Leftrightarrow G(1, T(x, y))=0 \Leftrightarrow$ $T(x, y)=0$.

3. $G(x, y)=1 \Leftrightarrow 2^{G(1, T(x, y))}-1=1 \Leftrightarrow$ $2^{G(1, T(x, y))}=2 \Leftrightarrow G(1, T(x, y))=1 \Leftrightarrow$ $T(x, y)=1 \Leftrightarrow x=y=1$.

4. $G$ is non-decreasing.

5. $G$ is continuous.

Theorem 7 Let $M$ be a continuous and increasing fucntion such that $M(x)=0 \Leftrightarrow x=0$ and $M(x)=1 \Leftrightarrow$ $x=1$. If $G_{T}$ is an overlap function and $T$ is a continuous t-norm, then

$$
G(x, y)=M\left(G_{T}(1, T(x, y))\right)
$$

is a t-overlap t-migrative function.
Theorem 8 Let $M$ be a n-dimensional function, nondecreasing, continuous such that $M\left(x_{1}, \ldots, x_{n}\right)=0 \Leftrightarrow$ $x_{i}=0$ for some $i \in 1, \ldots, n$ y $M\left(x_{1}, \ldots, x_{n}\right)=1 \Leftrightarrow$ $x_{i}=1$ for some $i \in 1, \ldots, n$. Then

$$
G(x, y)=M\left(G_{1}, \ldots, G_{n}\right)(1, T(x, y))
$$

is a t-overlap t-migrative function if $G_{i}$ is an overlap function for all $i \in 1, \ldots, n$ and continuous t-norm $T$.

\section{Proof 10 1. $G$ is symmetrical}

2. $G(x, y)=0 \Leftrightarrow M\left(G_{1}, \ldots, G_{n}\right)(1, T(x, y))=$ $0 \Leftrightarrow M\left(G_{1}(1, T(x, y)), \ldots, G_{n}(1, T(x, y))\right)=$ $0 \Leftrightarrow \exists k \in 1, \ldots, n$ such that $G_{k}(1, T(x, y))=$ $0 \Leftrightarrow T(x, y)=0$.

3. $G(x, y)=1 \Leftrightarrow M\left(G_{1}, \ldots, G_{n}\right)(1, T(x, y))=$ $1 \Leftrightarrow M\left(G_{1}(1, T(x, y)), \ldots, G_{n}(1, T(x, y))\right)=$ $1 \Leftrightarrow \exists k \in 1, \ldots, n$ such that $G_{k}(1, T(x, y))=$ $1 \Leftrightarrow T(x, y)=1 \Leftrightarrow x=y=1$.

4. $G$ is non-decreasing.

5. $G$ is continuous.

Theorem 9 Let $M$ be an n-dimensional and continuous function, such that $M\left(x_{1}, \ldots, x_{n}\right)=0 \Leftrightarrow x_{i}=0$ for some $i \in\{1, \ldots, n\}$ y $M\left(x_{1}, \ldots, x_{n}\right)=1 \Leftrightarrow x_{i}=1$ for some $i \in\{1, \ldots, n\}$. Then

$$
G(x, y)=M\left(G_{1}\left(1, T_{1}(x, y)\right), \ldots, G_{n}\left(1, T_{n}(x, y)\right)\right)
$$

is a t-overlap function with respect to some t-norm $T_{k}$ where $T_{i}$ are continuous t-norms and $G_{i}$ are overlap functions.

Theorem 10 A function $G_{S}:[0,1]^{2}-[0,1]$ is an overlap $t$-migrative function if and only if $G_{S}(x, y)=g(T(x, y))$ for all $x, y \in[0,1]$ holds for some non-decreasing function $g:[0,1]-[0,1]$ such that $g(0)=0$ y $g(1)=1$.

Proof 11 Let $G_{S}$ be an overlap t-migrative function, then there exists a non-decreasing function $g$ such that $G_{S}(x, y)=g(T(x, y))$. Now $g(0)=g(T(0,0))=$ $G_{S}(0,0)=0$. Besides $g(1)=g(T(1,1))=G_{S}(1,1)=$ 1 If $G_{S}(x, y)=g(T(x, y))$ then $G_{S}(x, T(y, z))=$ $G_{S}(T(x, T(y, z)))=g(T(T(x, y), z))=G_{S}(T(x, y), z)$.

Theorem 11 If $T$ is a continuous t-norm and $n$ is a strong negation then $G(x, y)=\frac{T(x, y)}{T(x, y)+n T(x, y)}$ is a t-overlap $t$ migrative function with respect to $T$.

Proof 12 By corollary it can be said that $G$ is a $t$ overlap function with respect to the t-norm $T$. On the other hand, $G(x, T(y, z))=\frac{T(x, T(y, z))}{T(x, T(y, z))+n T(x, T(y, z))}=$ $\frac{T(T(x, y), z)}{T(T(x, y), z)+n T(T(x, y), z)}=G(T(T(x, y), z))$. 
Theorem 12 Let $G_{1}, \ldots, G_{n}$ be t-migrative overlap functions with respect to T. If $\omega_{1}, \ldots, \omega_{n}$ are not negative real numbers such that $\sum_{i=1}^{n} \omega_{i}=1$ then

$$
G(x, y)=\sum_{i=1}^{n} \omega_{i} G_{i}(x, y)
$$

is a t-migrative overlap function with respect the $t$-norm $T$.

Proposition 2 If $G_{T}$ is a t-overlap function with respect to t-norm $T$, then $G_{T}$ is $t$-migrative with respect to $T$.

\section{Conclusions}

In this article we present a generalization of the concept of migrativity applied to overlap function, this generalization expands and allow the migrativity and the applications of the overlap functions on the topics about artificial intelligence and we present how we can generate new migrative functions.

\section{References}

Alcala-Fdez, J., Alcala, R., Herrera, F. (2011). A fuzzy association rule-based classification model for high-dimensional problems with genetic rule selection and lateral tuning. IEEE Transactions on Fuzzy Systems, 19(5), 857-872. https://doi.org/10.1109/ TFUZZ.2011.2147794
Bandler, W., Kohout, L. (1980). Fuzzy power sets and fuzzy implication operators. Fuzzy Sets and Systems, 4(1), 13-30. https://doi.org/10.1016/0165 $-0114(80) 90060-3$

Bustince, H., Fernandez, J., Mesiar, R., Montero, J., Orduna, R. (2010). Overlap functions. Nonlinear Analysis: Theory, Methods \& Applications, 72(3-4), 1488- 1499. https://doi.org/10.1016/j.na.2009.08.033

Bustince, H., Montero, J., Mesiar, R. (2009). Migrativity of aggregation functions. Fuzzy Sets and Systems, 160(6), 766-777. https://doi.org/10.1016/ j.fss. 2008.09.018

Durante, F., Sarkoci, P. (2008). A note on the convex combinations of triangular norms. Fuzzy Sets and Systems, 159(1), 77-80. https://doi.org/10.1016/ j.fss.2007.07.005

Fodor, J., Rudas, I. J. (2007). On continuous triangular norms that are migrative. Fuzzy Sets and Systems, 158(15), 1692-1697. https://doi.org/10.1016/ j.fss.2007.02.020

Grabisch, M., Marichal, J. L., Mesiar, R., Pap, E. (2009). Aggregation Functions. Encyclopedia of Mathematics and its Applications, Cambridge University, New York. https://doi.org/10.1017/CB09781139644150 